\title{
Field-tunable magnetic phases in a semiconductor-based two-dimensional Kondo lattice
}

\author{
C. Siegert ${ }^{\mathrm{a}}$, A. Ghosh ${ }^{\mathrm{b}}$, M. Pepper ${ }^{\mathrm{a}}$, I. Farrer ${ }^{\mathrm{a}}$, D. A. Ritchie ${ }^{\mathrm{a}}$, \\ D. Anderson ${ }^{\mathrm{a}}$, and G. A. C. Jones ${ }^{\mathrm{a}}$ \\ ${ }^{a}$ Cavendish Laboratory, University of Cambridge, J.J. Thomson Avenue, \\ Cambridge CB3 OHE, United Kingdom. \\ ${ }^{\mathrm{b}}$ Department of Physics, Indian Institute of Science, Bangalore 560 012, India.
}

\begin{abstract}
We show the existence of intrinsic localized spins in mesoscopic high-mobility GaAs/AlGaAs heterostructures. Non-equilibrium transport spectroscopy reveals a quasi-regular distribution of the spins, and indicates that the spins interact indirectly via the conduction electrons. The interaction between spins manifests in characteristic zero-bias anomaly near the Fermi energy, and indicates gate voltage-controllable magnetic phases in high-mobility heterostructures. To address this issue further, we have also designed electrostatically tunable Hall devices, that allow a probing of Hall characteristics at the active region of the mesoscopic devices. We show that the zero field Hall coefficient has an anomalous contribution, which can be attributed to scattering by the localized spins. The anomalous contribution can be destroyed by an increase in temperature, source drain bias, or field range.
\end{abstract}

Key words: Intrinsic spin lattice, 2DEG, Spin interaction, Anomalous Hall effect, RKKY

PACS: 72.25.-b, 71.45.Gm, 71.70.Ej

\section{Introduction}

Experimental simulations of fundamental magnetic interactions on few spin basis are very challenging. The most common approach investigates the interaction between localized spins with the spin cloud of surrounding conduction electrons, where localized spins are created by using either atomic nucleii [1], intentionally embedded magnetic impurities [2], or odd electron quantum dots (QDs) [3,4]. Individual localized spins can be employed to simulate 
the Kondo effect [3,4]. In systems containing more than one localized spin, the spins can interact via the Ruderman-Kittel-Kasuya-Yoshida (RKKY) exchange with interaction parameter $J^{\text {RKKY }}[5,6$. This has been shown experimentally for the two-spin case, where the interaction has been tuned electrically with surface gates [7]. The pairwise coupling can be ferromagnetic for $J^{\text {RKKY }}<0$, or anti-ferromagnetic for $J^{\text {RKKY }}>0$. For non-interacting spins, $J^{\text {RKKY }}=0$, the Kondo effect contributes one additional state at each localized spin within a bandwidth of $T_{\mathrm{K}}$ at $E_{\mathrm{F}}$. Non-equilibrium transport spectroscopy then shows a resonance in differential conductance $\mathrm{d} I / \mathrm{d} V$ at zero sourcedrain bias $\left(V_{\mathrm{SD}}=0\right)$ [8,9], which we call type-I zero bias anomaly (ZBA). With interacting spins $\left|J^{\mathrm{RKKY}}\right|>0$, the resonance is suppressed at $E_{\mathrm{F}}$ for $k_{\mathrm{B}} T<\left|J^{\mathrm{RKKY}}\right|$, and $\left|V_{\mathrm{SD}}\right|<\left|J^{\mathrm{RKKY}}\right|$, resulting in a split resonance, which we call type-II ZBA [10,11,12,13]. The half width at half maximum of the split is defined as $\Delta$ and acts as an indicator of spontaneous spin-polarization and static magnetic phases within the system [14,10,15].

Here we present high-mobility mesoscopic heterostructures that show signatures of an intrinsic spin lattice. As, in two dimensions, interspin distance $R$, and $k_{\mathrm{F}}=\sqrt{2 \pi n_{2 \mathrm{D}}}$ tunes the exchange interaction parameter $J^{\mathrm{RKKY}}\left(k_{\mathrm{F}}, R\right) \propto$ $\cos \left(2 k_{\mathrm{F}} R\right) / R^{2}$ for $k_{\mathrm{F}} R>>1$ [16,13], we can tune the magnetic state of the spin lattice with a simple non-magnetic surface gate. The interspin distance $R$ is fixed for a given device, and can be evaluated via phase interference effects at low temperatures, or with commensurability effects at temperatures $T>500 \mathrm{mK}$ [13]. Such a system is ideal for investigations of magnetic phases in two-dimensions. As non-equilibrium transport spectroscopy shows the magnitude $\left|J^{\text {RKKY }}\right|$ only, zero field Hall measurements have to be carried out that might enable the determination of the sign of $J^{\mathrm{RKKY}}$.

\section{Two probe transport spectroscopy}

Devices were fabricated from high mobility $\mathrm{Si}-\delta$-doped $\mathrm{GaAs} / \mathrm{Al}_{0.33} \mathrm{Ga}_{0.67} \mathrm{As}$ heterostructures. The 2DEG is formed $300 \mathrm{~nm}$ below the surface, and the spacer layer between dopants and the 2DEG is $80 \mathrm{~nm}$. Si doping density of $n_{\mathrm{Si}} \approx 2.5 \times 10^{12} \mathrm{~cm}^{-2}$ results in as-grown electron density $n_{2 \mathrm{D}} \approx 1 \times 10^{11} \mathrm{~cm}^{-2}$ with low temperature mobilities $\sim 1-3 \times 10^{6} \mathrm{~cm}^{2} / \mathrm{V}$-s. By selective wet-etching a $8 \mu \mathrm{m}$ wide mesa is created. One thermally deposited $2 \mu \mathrm{m}$ long non-magnetic $\mathrm{Ti} / \mathrm{Au}$ gate on the mesa is used to electrostatically vary $n_{2 \mathrm{D}} \approx 1-3 \times 10^{10} \mathrm{~cm}^{-2}$ in the active $2 \times 8 \mu \mathrm{m}^{2}$ region. Fig. 1(a) shows an optical microscope image of a typical device with electric setup.

The devices were characterized in dilution refrigerators with base temperatures down to $\approx 30 \mathrm{mK}$. Combined $\mathrm{AC}+\mathrm{DC}$ two-probe measurements with ac excitation voltage $V_{\mathrm{AC}} \ll k_{\mathrm{B}} T / e$ is used for non-equilibrium transport 


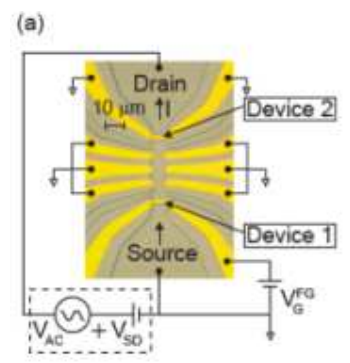

(c)

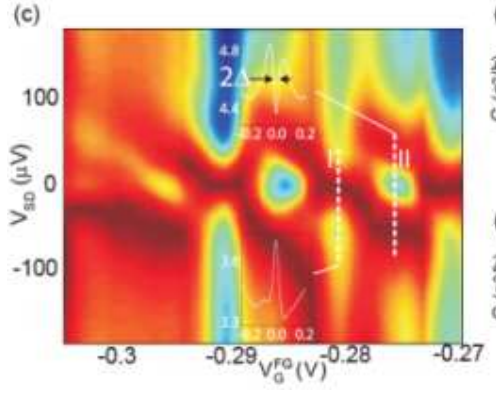

(b)

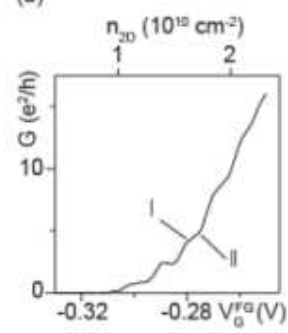

(d)

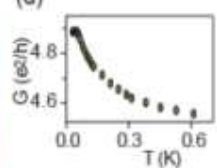

(e)

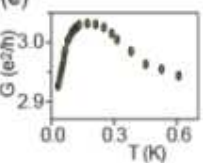

Fig. 1. (a) Optical image of typical device, including electrical connections. For operation, all gates are grounded with the exception of $V_{\mathrm{G}}^{\mathrm{FG}}$, which is used to vary the electron density in a $2 \times 8 \mu \mathrm{m}^{2}$ region. Mixed $\mathrm{AC}+\mathrm{DC}$ voltage is used to perform non-equilibrium transport spectroscopy. (b) At $35 \mathrm{mK}$ typical linear conductance $G\left(V_{\mathrm{G}}^{\mathrm{FG}}\right)$, and $n_{2 \mathrm{D}}\left(V_{\mathrm{G}}^{\mathrm{FG}}\right)$ in the upper panel. (c) Surface plot $\mathrm{d} I / \mathrm{d} V$ over $V_{\mathrm{G}}^{\mathrm{FG}}$ showing alternating type-I, and type-II ZBA. In the white insets typical type-I, and type-II ZBA are shown. (d) Typical temperature dependence of type-I ZBA showing the peak vanishes monotonically over $T$. (e) Typical temperature dependence of type-II ZBA showing non-monotonicity at $V_{\mathrm{SD}}=0$.

spectroscopy. In fig. 1(b) we show a typical linear conductance $G\left(V_{\mathrm{G}}^{\mathrm{FG}}\right)$, and $n_{2 \mathrm{D}}\left(V_{\mathrm{G}}^{\mathrm{FG}}\right)$ for $30 \mathrm{mK}$ at zero magnetic field. At temperatures $T<100 \mathrm{mK}$, $G\left(V_{\mathrm{G}}^{\mathrm{FG}}\right)$ shows characteristic features of type-I, and type-II ZBA. For most devices, the structures are strongest in the electron density range $n_{2 \mathrm{D}} \sim$ $1-3 \times 10^{10} \mathrm{~cm}^{-2}$, see fig. $1(\mathrm{~b})$, and often visible up to $G \gtrsim 10-15 \times\left(e^{2} / h\right)$. In non-equilibrium transport spectroscopy $\mathrm{d} I / \mathrm{d} V\left(V_{\mathrm{G}}^{\mathrm{FG}}, V_{\mathrm{SD}}\right)$ consists of a repetitive sequence of two-types of resonances as $V_{\mathrm{G}}$ is increased, see fig. 1(c). The single-peaked type-I ZBA splits intermittently to form a gap at $E_{\mathrm{F}}$, resulting in type-II ZBA. The insets (white) show the non-equilibrium traces of type-I $\mathrm{ZBA}$, and type-II ZBA at the $V_{\mathrm{G}}^{\mathrm{FG}}$ indicated in fig. $1(\mathrm{~b})$.

Type-I ZBA decrease monotonically with increasing T, see fig. 1(d), and with increasing parallel magnetic field $\mathrm{B}$ [9]. In non-equilibrium transport spectroscopy type-I ZBA split with $2 g \mu_{\mathrm{B}} B$ [13,9]. Type-II ZBA show a nonmonotonicity at the scale of $\Delta$ over both $\mathrm{T}$ (see fig. $1(\mathrm{e})$ ), and $\mathrm{B}$, which is characteristic for interacting spins $J^{\mathrm{RKKY}}>0$ [10,13,9]. Type-I ZBA can then be understood as a spin lattice with $\left|J^{\mathrm{RKKY}}\right| \ll k_{\mathrm{B}} T$. While the existence of mutually interacting localized spins in high-mobility mesoscopic heterostructures can account for such behavior, the question of spatial arrangement of the spins needs to be addressed independently with magnetoconductance mea- 

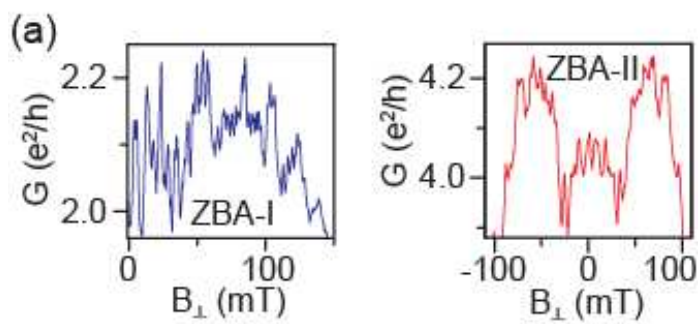

(b)

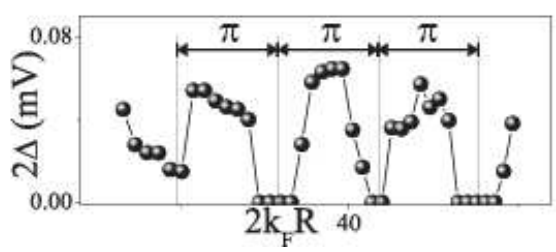

Fig. 2. (a) Low temperature $G\left(B_{\perp}\right)$ for type-I, and type-II ZBA showing Aharonov-Bohm like oscillations, which allow the determination of a regular lattice parameter R. (b) Using $\mathrm{R}$ from $\mathrm{AB}$-like oscillations, and $n_{2 \mathrm{D}}$ from capacitance model, $\Delta$ is found to be periodic in $\pi$ over $2 k_{\mathrm{F}} R$.

surements.

In two-dimensional spin lattice models, with localized spins arising from fluctuations in the conduction band, both Aharonov-Bohm like oscillations, as well as commensurability effects are expected [13]. Sites of localizes spins act as scattering centers, or as antidot, for conduction electrons, leading to backscattering and interference effects when the phase coherence length of electrons exceeds $R$. At low $T$, a random spatial distribution of the scatterers or antidots gives rise to weak localization phenomena. The magnetoconductance $G\left(B_{\perp}\right)$ then shows universal aperiodic fluctuations. However, for a quasi-regular antidot array, transport can be modeled through multiple, connected AharonovBohm rings encircling a discrete number of impurities. Aharonov-Bohm like oscillations with periodicity $\Delta B_{\perp} \approx h / \pi e R_{\mathrm{C}}^{2}$, where $R_{\mathrm{C}}$ is the radius of a stable ring [17 18], appear. The oscillations can be assigned to the respective orbits via Fourier Transform, and the lowest possible orbit gives an estimate for $R[13$. Fig. 2(a) shows typical oscillations in the magnetoconductance for a type-I, and a type-II ZBA. We cross-checked the quasi regular distribution of the localized spins at higher $T$ from commensurability effects of classical electron trajectories in a 2D array of point scatterers at finite $B_{\perp}[19] 13$. Please note, that the magnetoconductance oscillations appear only over a range of $n_{2 \mathrm{D}}$, where ZBA is observed.

Associating each antidot to one (or more) localized spin then realizes a spin array embedded in the sea of conduction electrons [13]. These spins can interact via the conduction electrons by RKKY mechanism, and are then expected to have a characteristic periodicity of $\delta\left(2 k_{\mathrm{F}} R\right)=\pi$ in $\left|J^{\mathrm{RKKY}}\right|$. As $\Delta \propto\left|J^{\mathrm{RKKY}}\right|$, we can confirm this by plotting in fig. $2(\mathrm{~b}) \Delta\left(2 k_{\mathrm{F}} R\right)$. The clear periodicity of $\approx \pi$ is recognized as the for RKKY typical $2 k_{\mathrm{F}} R$-oscillations, establishing the 

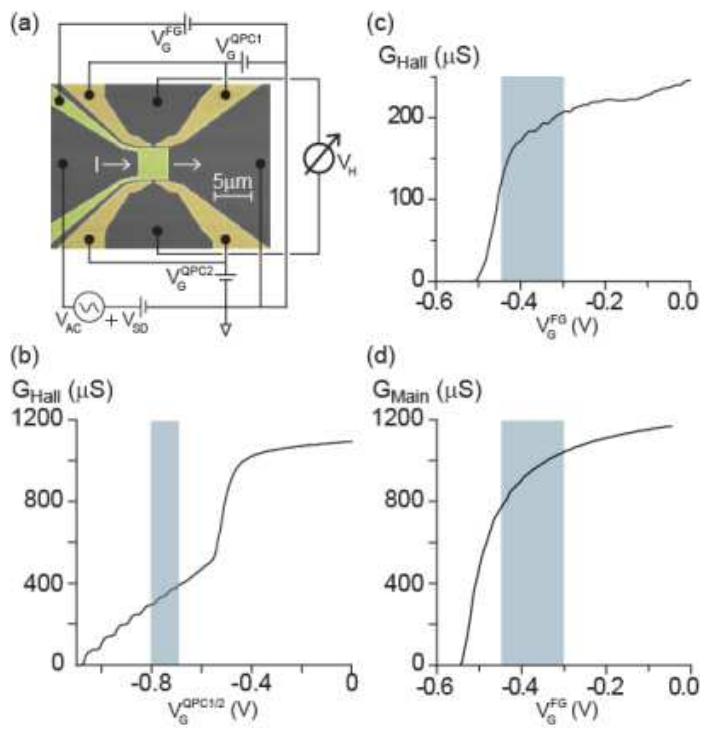

Fig. 3. (a) Optical image of a $4 \times 4 \mu \mathrm{m}^{2}$ Hall device. $V_{\mathrm{G}}^{\mathrm{QPC} 1}$, and $V_{\mathrm{G}}^{\mathrm{QPC} 1}$ define a $4 \mu \mathrm{m}$ wide mesa with QPC to probe Hall voltage. $V_{\mathrm{G}}^{\mathrm{FG}}$ varies $n_{2 \mathrm{D}}$ in the active $4 \times 4 \mu \mathrm{m}^{2}$ region. (b) Voltages $V_{\mathrm{G}}^{\mathrm{QPC} 1 / 2}$ are operated between $-0.8 \mathrm{~V}$ and $-0.7 \mathrm{~V}$ to ensure open channel with strong confinement. (c) For both $\mathrm{QPC}$ voltages $V_{\mathrm{G}}^{\mathrm{QPC} 1 / 2}=-0.8 \mathrm{~V}$, the full gate $V_{\mathrm{G}}^{\mathrm{FG}}$ is operated such that the conductance in Hall direction does not drop below $2 e^{2} / h$ (grayish region). (d) For same conditions conduction in the main direction, as used for the experiment.

existence of an interacting spin lattice in high mobility heterostructures [13].

\section{Zero field Hall coefficient}

While non-equilibrium transport spectroscopy provides an indirect evidence of localized spins in high-mobility heterostructures, a convenient, and more direct, mean to probe the magnetic state of a two-dimensional spin lattice is the initial, or zero field, Hall coefficient. In case of magnetic order, the finite magnetization of the system adds an additional term to the Hall constant, resulting in the anomalous Hall effect (AHE) [20]. The Hall resistance is then $R_{\mathrm{H}}=\gamma_{\mathrm{H}}^{0} B_{\perp}+\gamma_{\mathrm{A}} M$, with $B_{\perp}$ being magnetic field, M being magnetization, $\gamma_{\mathrm{H}}^{0}=-1 / n_{2 \mathrm{D}}|e|$ in two dimensions, and $\gamma_{\mathrm{A}}$ being the anomalous Hall constant. We have designed fully tunable Hall devices, and show in fig. 3(a) an optical image with electrical setup. The 2DEG is laterally confined by two pairs of nonmagnetic $\mathrm{Ti} / \mathrm{Au}$ sidegates with voltages $\mathrm{V}_{\mathrm{G}}^{\mathrm{QPC} 1}$ and $\mathrm{V}_{\mathrm{G}}^{\mathrm{QPC} 2}$ between $0.7 \mathrm{~V}$ and $-0.8 \mathrm{~V}$, which ensures defined QPCs, combined with low resistance Hall probes. Fig. 3(b) shows typical $G_{\text {Hall }}\left(V_{\mathrm{G}}^{\mathrm{QPC}}\right)$ for the QPCs. The voltages $\mathrm{V}_{\mathrm{G}}^{\mathrm{QPC} 1}$ and $\mathrm{V}_{\mathrm{G}}^{\mathrm{QPC} 2}$ are adjusted at the beginning of the measurement, and kept constant during the whole experiment. The resulting 4-5 $\mu \mathrm{m}$ wide electrostatically defined mesa is patterned with a full $\mathrm{Ti} / \mathrm{Au}$ gate of dimensions $4 \times 4$ 

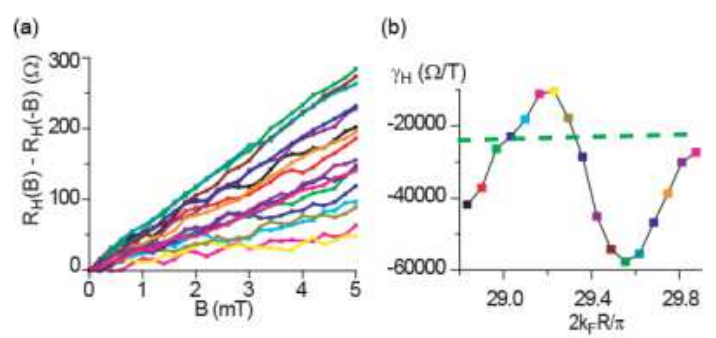

Fig. 4. (a) Asymmetric Hall resistance $\mathrm{R}_{\mathrm{H}}=\frac{1}{2 I}\left(V_{\mathrm{H}}\left(B_{\perp}\right)-V_{\mathrm{H}}\left(-B_{\perp}\right)\right)$ up to $5 \mathrm{mT}$, with different colors indicating different electron densities. (b) The respective Hall coefficients $\gamma_{\mathrm{H}}$ over $2 k_{\mathrm{F}} R / \pi$ showing a strong oscillation. The high temperature, undistorted $\gamma_{\mathrm{H}}$ is shown as dashed green line.

$\mu \mathrm{m}^{2}$ or $5 \times 5 \mu \mathrm{m}^{2}$ in between the two side gates. Voltage on the full gate $\mathrm{V}_{\mathrm{G}}^{\mathrm{FG}}$ is applied to vary $E_{\mathrm{F}}$ and thus create the active part of the device, with $\mathrm{n}_{2 \mathrm{D}}=2-5 \times 10^{10} \mathrm{~cm}^{-2}$. In fig. $3(\mathrm{c})$ we show $G_{\text {Hall }}\left(V_{\mathrm{G}}^{\mathrm{FG}}\right)$ for $-0.8 \mathrm{~V}$ at the QPCs, and the range in which $V_{\mathrm{G}}^{\mathrm{FG}}$ is operated in gray, which is chosen to ensure $\mathrm{G}_{\text {Hall }} \gg 2 \frac{e^{2}}{h}$ for all $\mathrm{V}_{\mathrm{G}}^{\mathrm{FG}}$ used.

With $\mathrm{V}_{\mathrm{G}}^{\mathrm{QPC} 1}$ and $\mathrm{V}_{\mathrm{G}}^{\mathrm{QPC} 2}$ both set at $-0.8 \mathrm{~V}$, the conductance $G$ in the longitudinal direction was maintained at $G>10 e^{2} / h$ for all $V_{\mathrm{G}}^{\mathrm{FG}}$ when the device was in operation for the Hall measurements. Please note that $\mathrm{n}_{2 \mathrm{D}}$ and $G$ are higher in this experiment than in earlier ones to ensure free paths both in longitudinal, as well as in Hall direction. The electron density is determined at high $\mathrm{n}_{2 \mathrm{D}}$ in the range of $\pm 50 \mathrm{mT}$, where no fluctuations are visible, and both low, and high $\mathrm{T}$ result in the same electron density (see inset of fig. 5). Lower electron densities were determined by extrapolating the dependence of $n_{2 \mathrm{D}}$ on $V_{\mathrm{G}}$ at the large $n_{2 \mathrm{D}}$ regime via standard capacitance model.

The samples are measured in a dilution refrigerator at base electron temperature $T \approx 75 \mathrm{mK}$. Two probe low frequency $(\approx 13 \mathrm{~Hz})$ transport measurement with $\mathrm{AC}$ voltage $\mathrm{V}_{\mathrm{AC}} \ll k_{\mathrm{B}} T / e$, and source-drain bias $\mathrm{V}_{\mathrm{SD}}$ is carried out such that current flows in the main direction through the active part only. Perpendicular magnetic field $\mathrm{B}_{\perp}$ is applied to measure the initial Hall coefficient $\gamma_{\mathrm{H}}$, taken from the slope of the asymmetric Hall resistance $\mathrm{R}_{\mathrm{H}}=\frac{1}{2 I}\left(V_{\mathrm{H}}\left(B_{\perp}\right)-V_{\mathrm{H}}\left(-B_{\perp}\right)\right)$, with $I$ being the current, and $V_{\mathrm{H}}\left(B_{\perp}\right)$ being the Hall voltage on the QPCs, over the initial 2-5 mT. The asymmetric Hall resistance is taken only, to cancel out misalignment of Hall probes, and other field symmetric artifacts [21]. In fig. 4(a) we show the asymmetric component of the Hall resistance up to $5 \mathrm{mT}$, with different colors belonging to different electron densities. Via Aharonov-Bohm-type oscillations (Fig. 2) we find the interspin distance $\mathrm{R} \approx 1.1 \mu \mathrm{m}$ in these devices. The slope of the Hall resistance gives the Hall coefficient, which we show for the respective electron densities in fig. 4(b). The dashed green line indicates the unperturbed value of $\gamma_{\mathrm{H}}$, and $\gamma_{\mathrm{H}}$ shows a strong deviation from its high temperature value. 


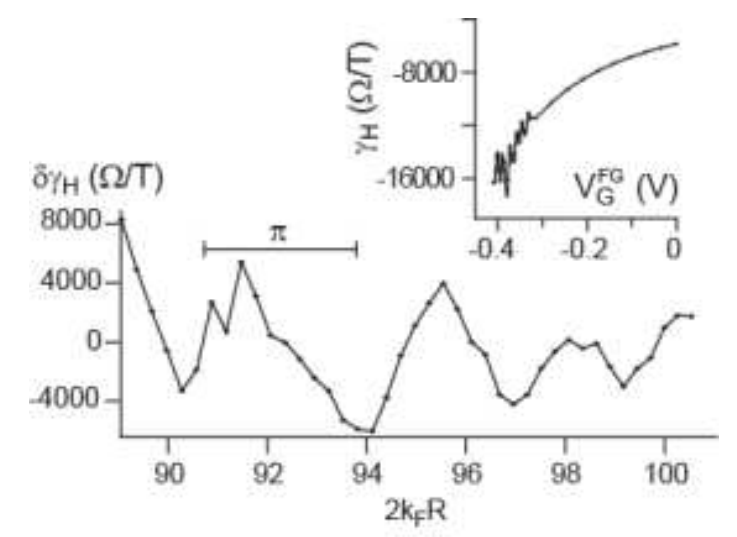

Fig. 5. The deviation $\delta \gamma_{\mathrm{H}}=\gamma_{\mathrm{H}}-\gamma_{\mathrm{H}}^{0}$ over a larger range of $2 k_{\mathrm{F}} R$ shows periodic fluctuations with both positive, and negative sign, and periodicity of $\pi$. Inset: Hall coefficient at high density, and high field range, as used for calculation of electron density.

The Hall coefficient deviates both positively and negatively with respect to the unperturbed value $\gamma_{\mathrm{H}}^{0}$. The positive contribution to $\gamma_{H}$ is unexpected, and has the wrong sign for a magnetic contribution via the anomalous Hall effect. The negative deviation is expected for a magnetic interaction in a spin lattice. To investigate that further, we have considered a larger range of densities, and show in fig. 5 the range $2 k_{\mathrm{F}} R=89-101$. For clarity, only the deviation $\delta \gamma_{\mathrm{H}}=\gamma_{\mathrm{H}}-\gamma_{\mathrm{H}}^{0}$ is plotted. For a given period in $2 k_{\mathrm{F}} R$, the deviation $\delta \gamma_{\mathrm{H}}$ again is either positive or negative, and overall, the magnitude of this deviation decreases when the carrier density is increased. This can be due to the screening of localized spins, which increases with increasing electron density, and reduces the visibility of the localized spins. $\delta \gamma_{\mathrm{H}}$ has a rough periodicity of $\delta\left(2 k_{\mathrm{F}} R\right)=\pi$, indicating a common origin of these fluctuations and the ZBA in nonequilibrium transport.

Please note that clean periodicity of fluctuations in $\gamma_{\mathrm{H}}$ requires the whole device area to be in the same phase. Since the formation of the intrinsic spin lattices does not yield perfect regularity in interspin distance $R$ over the whole $4 \times 4 \mu \mathrm{m}^{2}$ or $5 \times 5 \mu \mathrm{m}^{2}$ in $100 \%$ of our devices, we do also observe random fluctuations, and show in fig. 6c) also a bad device.

\subsection{Stability of fluctuations}

In fig. 6(a) we show a typical fluctuation of $\gamma_{\mathrm{H}}$ for various source drain biases. For all other experiments $V_{\mathrm{SD}}=0$ was kept constant. The fluctuation vanishes with increasing $V_{\mathrm{SD}}$, and is back to its unperturbed value $\gamma_{\mathrm{H}}^{0}$ (shown as dashed green line) at about $V_{\mathrm{SD}} \approx 400 \mu \mathrm{V}$.

In fig. 6(b) we show the deviation of $\gamma_{\mathrm{H}}$, calculated from slope of the asymmet- 


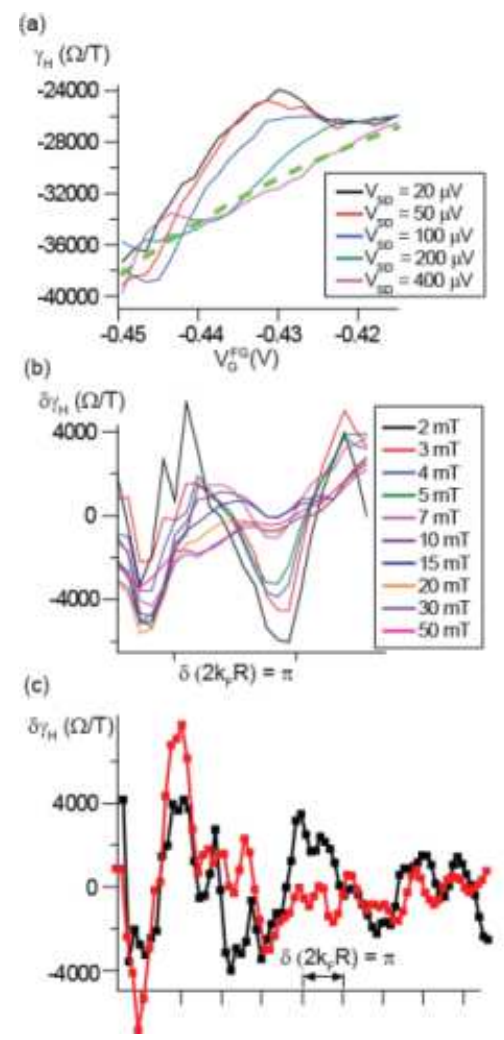

Fig. 6. (a) Non-equilibrium $\gamma_{\mathrm{H}}$, where application of $V_{\mathrm{SD}}$ destroys the fluctuations in $\gamma_{\mathrm{H}}$. The dashed green line indicates the extrapolated $\gamma_{\mathrm{H}}$ from capacitance model. (b) $\delta \gamma_{\mathrm{H}}$ taken with various field ranges. The fluctuations in $\gamma_{\mathrm{H}}$ vanish for higher field ranges. (See text) (c) $\delta \gamma_{\mathrm{H}}$ at low $n_{2 \mathrm{D}}$ showing the effect of disorder, and only few regular fluctuations. The black trace is for confinement voltage of $-0.8 \mathrm{~V}$, and the red trace is taken with $-0.7 \mathrm{~V}$.

ric Hall resistance in various B field ranges, from $\gamma_{\mathrm{H}}^{0}$. The lowest field ranges show strongest fluctuations, and increasing field range decreases the intensity of the fluctuations. Since the interspin interaction itself couples to very small transverse fields [22], the fluctuations are only meaningful when the slope is computed over smallest field ranges, i.e., leading to the zero field Hall coefficient. Since very low field ranges do also increase the error in the data, we use 2-5 $\mathrm{mT}$ range for all $\gamma_{\mathrm{H}}$ data. We find that the highest field range $50 \mathrm{mT}$ is mostly similar to the unperturbed Hall coefficient evaluated from the high density range and capacitance model.

By increasing the temperature the fluctuations in $\gamma_{\mathrm{H}}$ vanish (not shown), confirming the oscillations in Hall coefficient to be a low $T$ effect.

As mentioned before, disorder appears to be a crucial aspect of the fluctuations in $\gamma_{\mathrm{H}}$. We therefore show in fig. 6(c) an example of a bad sample with strong disorder, and mostly irregular fluctuations in $\delta \gamma_{\mathrm{H}}$. While no periodicity over the whole range can be seen, most adjacent fluctuations are still showing a rough $\pi$-periodicity, although not as clear as the less disordered devices. While 
the overall magnitude of fluctuations is increasing with lower densities, we find that the clean regularity of the fluctuations is decreasing with decreasing $n_{2 \mathrm{D}}$, where more random scattering appears. To avoid this we now record data at higher electron densities only, and find much cleaner $\pi$-periodic fluctuations, with only very little disorder.

The role of the confinement voltage is also shown in fig. 6(c). The red trace is shown for a confinement voltage of $-0.7 \mathrm{~V}$, and the black one for $-0.8 \mathrm{~V}$. The fluctuations in $\gamma_{\mathrm{H}}$ change the overall appearance with change in gate voltage, but not the characteristics. This is a very important point to note, as the state of the device should only weakly depend on the confinement voltage. When comparing the red and black traces, then minima, and maxima of $\delta \gamma_{\mathrm{H}}$ are untouched by the confinement voltage.

An important question to be addressed is the origin of the localized spins. While a model with localized spins arising from doping was suggested and analysed earlier [13], other origins can not be excluded yet. The interface between GaAs/AlGaAs could potentially induce potential fluctuations in the 2DEG. In our system the lattice constants are $a_{\mathrm{GaAs}}=0.565325 \mathrm{~nm}$ and $a_{\mathrm{Al}_{0.33} \mathrm{Ga}_{0.67} \mathrm{As}}=0.56559 \mathrm{~nm}$, resulting in a lattice mismatch of $\Delta a=0.047 \%$, and an average defect distance of $\approx 2 \mu \mathrm{m}$. This is close to the length scale we observe, but the defects are expected to be in stripes, not in puddles, as observed in our measurements. A purely electrostatic origin for the spin lattice might also cause such ZBA, but it is difficult to imagine how the large length scale arises. Currently, experiments are carried out that will enable a more detailed insight into the origin of the localized spins [23].

\section{Summary}

We report existence of an intrinsic spin lattice in high mobility GaAs/AlGaAs heterostructures. The localized spins interact indirectly via RKKY interaction, and the magnetic state of the lattice can be tuned with a simple non-magnetic surface gate. The interspin distance can be evaluated with Aharonov-Bohm like interference at low temperatures, and commensurability effects at higher temperatures. To probe the magnetic state directly, a Hall geometry is introduced, and existence of fluctuations in the Hall coefficient $\gamma_{\mathrm{H}}$ shown. The fluctuations are periodic in $2 \delta\left(k_{\mathrm{F}} R\right)=\pi$, and deviate positively and negatively from the non-interacting value $\gamma_{\mathrm{H}}^{0}$. 


\section{Acknowledgment}

This project was supported by EPSRC. CS acknowledges the support of Cambridge European Trust, EPSRC, and Gottlieb Daimler and Karl Benz Foundation.

\section{References}

[1] J. H. Smet, R. A. Deutschmann, F. Ertl, W. Wegscheider, G. Abstreiter, and K. von Klitzing, Nature 415, 281 (2002).

[2] V. Madhavan, W. Chen, T. Jamneala, M. F. Crommie, and N. S. Wingreen, Science 280, 567 (1998).

[3] D. Goldhaber-Gordon, H. Shtrikman, D. Mahalu, D. Abusch-Magder, U. Meirav, and M. A. Kastner, Nature 391, 156 (1998).

[4] S. M. Cronenwett, T. H. Oosterkamp, and L. P. Kouwenhoven, Science 281, 540 (1998).

[5] C. Jayaprakash, H.R. Krishnamurthy, and J.W. Wilkins, Phys. Rev. Lett. 47, 737 (1981).

[6] Affleck, I. \& Ludwig, A. W. W. Exact critical theory of the two-impurity Kondo model. Phys. Rev. Lett. 68, 1046 (1992).

[7] N. J. Craig, J. M. Taylor, E. A. Lester, C. M. Marcus, M. P. Hanson, and A. C. Gossard, Science 304, 565 (2004).

[8] L. Kouwenhoven and L. Glazman, Physics World January (2001).

[9] A. Ghosh, M. H. Wright, C. Siegert, M. Pepper, I. Farrer, C. J. B. Ford, and D. A. Ritchie, Phys. Rev. Lett. 95, 066603 (2005).

[10] H. B. Heersche, Z. de Groot, J. A. Folk, L. P. Kouwenhoven, H. S. J. van der Zant, A. A. Houck, J. Labaziewicz, and I. L. Chuang, Phys. Rev. Lett. 96, 017205 (2006).

[11] W. G. van der Wiel, S. De Franceschi, J. M. Elzerman, S. Tarucha, L. P. Kouwenhoven, J. Motohisa, F. Nakajima, and T. Fukui, Phys. Rev. Lett. 88, 126803 (2002).

[12] A. Ghosh, C. J. B. Ford, M. Pepper, H. E. Beere, and D. A. Ritchie, Phys. Rev. Lett. 92, 116601 (2004).

[13] C. Siegert, A. Ghosh, M. Pepper, I. Farrer, and D. A. Ritchie, Nature Physics 3, 315 (2007).

[14] A.N. Pasupathy, R.C. Bialczak, J. Martinek, J.E. Grose, L.A.K. Donev, P.L. McEuen, and D.C. Ralph, Science 306, 86 (2004). 
[15] J. Nygard, D.H. Cobden, and P.E. Lindelof, Nature 408, 342 (2000).

[16] M. T. Béal-Monod, Phys. Rev. B 36, 8835 (1987).

[17] R. Schuster, K. Ensslin, D. Wharam, S. Khn, and J.P. Kotthaus, Phys. Rev. B 49, 8510 (1994).

[18] Weiss, D. et al. Phys. Rev. Lett. 70, 4118 (1993).

[19] Weiss, D. et al. Phys. Rev. Lett. 66, 2790 (1991).

[20] H. Toyosaki, T. Fukumura, Y. Yamada, K. Nakajima, T. Chikyow, T. Hasegawa, H. Koinuma, and M. Kawasaki, Nat Mater 3, 221 (2004).

[21] W. Teizer, F. Hellman, and R. C. Dynes, Phys. Rev. B 67, 121102 (2003).

[22] C. Siegert, A. Ghosh, M. Pepper, I. Farrer, D. A. Ritchie, D. Anderson, G. A. C. Jones, submitted to PRL.

[23] S. Sarkozy et al., to be published 